\title{
Boosting Detection Sensitivity by Using a Surface-Wave-Enabled Darkfield Aperture (SWEDA)
}

\author{
Guoan Zheng**a Samuel Yang ${ }^{\mathrm{a}}$ and Changhuei Yang ${ }^{\mathrm{a}, \mathrm{b}}$ \\ ${ }^{a}$ Electrical Engineering, California Institute of Technology, Pasadena, CA 91125, USA \\ ${ }^{\mathrm{b}}$ Bioengineering, California Institute of Technology, Pasadena, CA 91125, USA \\ *gazheng@,caltech.edu
}

\begin{abstract}
The on-chip detection of a weak optical signal in biological experiments can easily be complicated by the presence of an overwhelming background signal, and as such, pre-detection background suppression is substantively important for weak signal detection. In this paper, we report a structure that can be directly incorporated onto optical sensors to accomplish background suppression prior to detection. This structure, termed surface-wave-enabled darkfield aperture (SWEDA), consists of a central sub-wavelength hole surrounded by concentric grooves that are milled onto a gold layer. Incoming light can be collected and converted into surface waves (SW) by the concentric grooves and then be recoupled into propagating light through the central hole. We show that the SW-assisted optical component and the direct transmission component of the central hole can cancel each other, resulting in near-zero transmission under uniform illumination (observed suppression factor of 1230). This structure can therefore be used to suppress a light field's bright background and allow sensitive detection of localized light field non-uniformity (observed image contrast enhancement of $27 \mathrm{~dB}$ ). We also show that under a coherent background illumination, a CMOS pixel patterned with the proposed structure achieves better SNR performance than an un-patterned single pixel.
\end{abstract}

Keywords: Surface plasmon, optical transfer function, darkfield imaging, signal to noise ratio

\section{INTRODUCTION}

Miniaturization and integration are two key features of device engineering in our time. The use of complementary metal-oxide-semiconductor (CMOS) integrated circuit (IC) technology for biological and biomedical applications is a good example of this engineering trend. A variety of CMOS chip-based analytical instruments have been developed in the past years, such as an optofluidic microscope [1,2], a magnetic cell manipulation system [3], a microarray platform for DNA and protein analysis and diagnostics [4], a wide field of view microscope [5], and microelectrode arrays for monitoring cell activity [6]. In contrast with conventional clinical platforms, which are bulky with high power consumption, CMOS chip-based instruments meet the growing need for hand-held, miniaturized, and highly automated devices for point-of-care biological testing. Among the various applications of the CMOS IC instruments, one major category is on-chip sensing and imaging systems based on a CMOS image sensor. Coupled to a microfluidic platform for sample delivery and confinement, CMOS image sensors offer the promise of achieving higher speed, lower power consumption, and higher-level integration in a portable lab-on-chip format. However, the detection of a weak optical signal using a CMOS image sensor can be easily confronted by an overwhelming background. The case of stars in the sky provides a good illustration of some of these limitations. A bright star that is quite visible at night may disappear from our sight during the day. This disappearing act is attributable to two major factors. First, the bright daytime background can introduce a proportionate noise term that the brightness of the star must exceed in order to be observable. Second, our eyes naturally adjust their dynamic range to accommodate the bright daytime background. As the bit depths of most measurement systems (including our eyes) are finite, we necessarily view the sky with a coarser brightness scale during the day. If the incremental brightness of the star versus the background is smaller than this gradation scale, the star is simply indistinguishable from its background. The approach of adding bit depth can address part of the problem; however, it is an 'engineering' solution that comes at the price of more sophisticated electronics and greater data volume. Moreover, it does not eliminate the proportionate noise term from the background. Interference arrangements can potentially be employed to destructively interfere and cancel the background (for situations where the light sources involved are coherent). However, such schemes are understandably elaborate and non-trivial to employ. A

Plasmonics in Biology and Medicine VIII, edited by Tuan Vo-Dinh, Joseph R. Lakowicz,

Proc. of SPIE Vol. 7911, 79110X · (C) 2011 SPIE · CCC code: 1605-7422/11/\$18 · doi: 10.1117/12.871044

Proc. of SPIE Vol. $791179110 X-1$ 
sensor that can intrinsically cancel a strong background prior to signal detection would be a simpler solution with broad applicability.

In this manuscript, we will describe the operating principle of the proposed structure. Then, we will report on our simulations and experimental implementation of two types of SWEDA (one is based on linear grooves and the other one is based on circular grooves). We next report on our experimental demonstration of the ability of the circular-groovebased SWEDA to detect weak signals in the presence of a strong background; we also present a proof-of-concept that demonstrates that sensors incorporating such structures can be used to implement a new class of darkfield microscopes, amongst other applications. Finally, we report on the successful implementation of a surface-wave-enabled dark-field aperture directly on a CMOS sensor pixel (Aptina MT9P031, with a $2.2 \mu \mathrm{m}$ pixel size).

\section{WORKING PRINCIPLE OF SWEDA}

The light interaction between the subwavelength features on a metal-dielectric interface is opening up exciting new opportunities in applications ranging from subwavelength optics to chemical sensing and biophysics [7-24]. It has been shown that appropriately patterned rings of metal corrugation around a hole can significantly change the total amount of light transmission through the aperture $[8,10,11,15,16,18,20]$. One primary component involved in such a lightinteraction between the central hole and the metal corrugation is the surface plasmon (SP) wave, the electromagnetic surface wave existing at the interface between a dielectric and a noble metal [25]. The SP wave has a wave vector of $\mathrm{k}_{\mathrm{s}}=\mathrm{k}_{0} \sqrt{\frac{\varepsilon_{\mathrm{m}} \varepsilon_{\mathrm{d}}}{\varepsilon_{\mathrm{m}}+\varepsilon_{\mathrm{m}}}}$, where $\mathrm{k}_{0}$ is the free space wave vector, and $\varepsilon_{\mathrm{m}}$ and $\varepsilon_{\mathrm{d}}$ are the permittivity of the metal and dielectric. SP waves exhibit intrinsic field localization at the interface and thus allow for the manipulation of light in the subwavelength scale. However, the SP wave is not the only component involved in the light-interaction of subwavelength features on the metal-dielectric interface. Recently, some theoretical and experimental results [11, 14-17, $21,23]$ show that a surface scattered component also plays a role at the short range interaction. Therefore, the mediatedtransmission behavior of this corrugation based aperture can be intuitively explained as follows. When light falls on a patterned groove structure on the metal, it couples into the surface wave (SW), including the SP wave and the surface scattered wave. By choosing the groove periodicity such that the surface wave launched at each groove adds up in phase, we can generate a strong propagative surface wave that is directed towards the hole. The surface wave can then be converted back to a propagating optical wave at the central hole. In essence, the groove structure serves as an antenna for light collection and uses the surface wave to transport the collected optical power to the hole. Using this approach, researchers have reported both light transmission enhancement and suppression $[8,10,11,15,18,26]$.
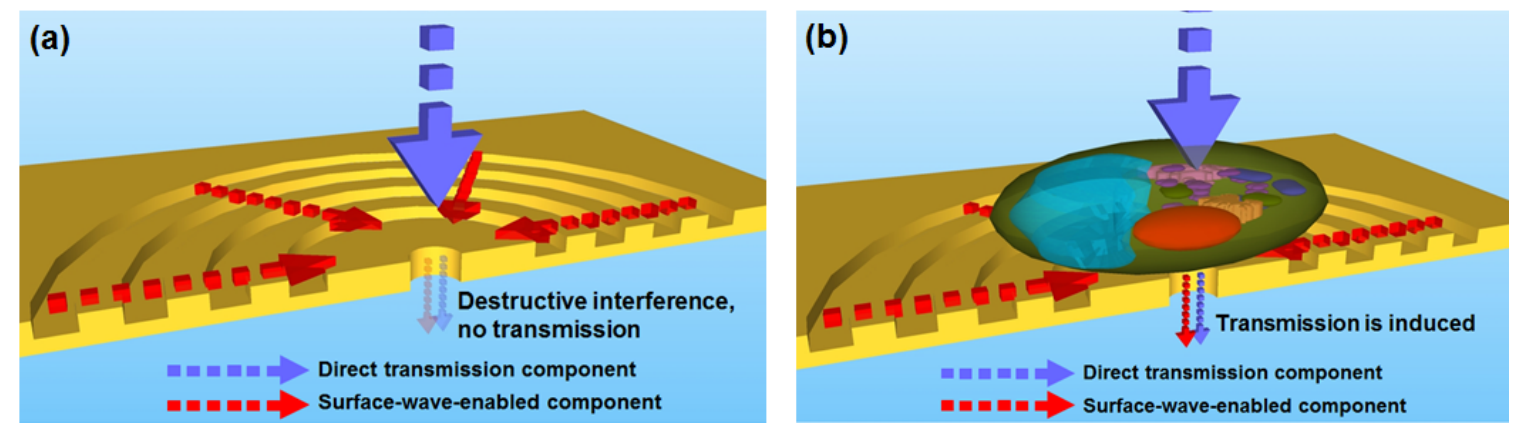

Figure 1. (a) The destructive interference between the direct transmission component and the surface-waveenabled component results in zero transmission. (b) A cell attached to the surface of SWEDA will break the destructive interference condition and induce transmission.

Our SWEDA design [27] differs from previous surface-wave-modulated apertures by exactly balancing the direct transmission component of the central hole and the surface wave component induced from the grooves. We precisely control the amplitude and the phase of the surface wave by changing the periodicity, depth of the groove structure and spacing between the central hole and groove. Through judicious choice of these parameters, we can arrive at a situation where the two components will destructively interfere and result in little or no light transmission through the hole in the presence of uniform normal-incidence illumination (Fig. 1(a)). Since this destructive interference condition critically depends on an exact balance of the two mentioned components, a small change in spatial distribution of the input light 
field intensity or phase will disrupt the destructive interference condition and permit significant light transmission through the hole (Fig. 1(b)).

In the context of high sensitivity optical signal detection, the advantage of SWEDA can be easily appreciated. The structure can effectively suppress a uniform background from reaching the underlying sensor and instead only permit highly localized light field variations to pass through and be detected. As such, the underlying sensor no longer needs to contend with the high background and its associated noise fluctuation terms. The bit depth can also be optimized and devoted to the detection of the weaker light field variations. Used in an appropriate manner, such devices can potentially allow for greater signal detection sensitivity in weak-signal-buried-in-high-background scenarios. This method also enables a new way to build darkfield microscopes on the sensor level that does not rely on elaborate and bulky optical arrangements.

\section{SWEDA WITH LINEAR GROOVE PATTERN}

Fig. 2(a) and 2(b) show the working principle of the proposed SWEDA with a linear groove pattern. Incoming TM polarized light (where the electric-field is perpendicular to the groove structure) can be collected and converted into the surface wave by the periodic grooves and then recoupled into propagating light through the central hole. We show that such SW-assisted components and the direct transmission component of the central hole can cancel each other, resulting in near-zero transmission under uniform illumination (Fig. 2(a)). On the other hand, the SP coupling efficiency for TE polarized light (where the magnetic-field is perpendicular to the groove structure) is much smaller than that for the TM wave [25], and thus, the absence of interference of SP components permits significant TE polarized light transmission through the hole in Fig. 2(b). We perform a set of simulations to understand the interplay between our design parameter choices and system characteristics by using a commercial simulation software - CST Microwave Studio [28]. The simulations were performed at a nominal wavelength of $750 \mathrm{~nm}$. The permittivity of gold at this wavelength is $-20.96+$ 1.55i [29]. There are 4 specific parameters that impact the SWEDA's performance; they are: 1) Groove periodicity. The groove periodicity (defined here as the p-parameter in Fig. 1C) can be adjusted based on the SP dispersion relationship to control the magnitude of the SP-wave coupled into the structure. Note that the exact match of the groove periodicity to the SP wavelength is not necessarily desired, as this may induce an overly strong SP-wave component, which cannot be matched by the direct transmission component. 2) The number of grooves. The strength of the coupled SP wave increases as a function of the number of grooves. On the other hand, we desire a low number of grooves for overall SWEDA structure compactness considerations. 3) Central hole size. This affects the strength of the direct transmission component. We would additionally want to restrict the aperture size such that the light transmission is not multi-moded. Multi-mode light transmission significantly complicates our destructive interference balancing act as we would need to achieve destructive interference between the SP-assisted component and the direct transmission component for all modes involved. 4) The distance between the innermost groove and the rim of the central hole (defined here as the s-parameter in Fig. 2(a)) determines the phase difference between the SP-assisted and the direct transmission components. To accomplish exact cancellation of the two components, we require this phase difference to be 180 degrees.

The simulation program allowed us to map out the interplay of these parameters and the overall SWEDA system characteristics. We define the darkfield suppression factor as the ratio of the total power transmission through a simple hole (without grooves) to the total power through a SWEDA. For good darkfield performance, we desire this ratio to be as high as possible. We were able to arrive at a design parameter set that provides a suppression factor of 35400 in our simulations. The simulated magnetic-field distributions for this particular SWEDA design and that of a corresponding simple hole are shown in Fig. 2(a) and 2(c). We can see that the SWEDA structure should indeed be able to suppress light transmission through the central hole significantly. In Fig. 2(b), we also show the electric-field distributions for the TE wave, where significant transmission is induced. The difference between the TM and TE cases also verifies the SPenabled mechanism of the SWEDA, since the SP wave can only be induced for TM polarization [25]. We also note that, from the simulations shown in Fig. 2(a) and 2(b), the SWEDA provides a polarization extinction ratio of 42000 for the two orthogonal polarization states. 
(a)

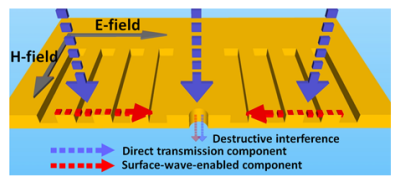

(b)

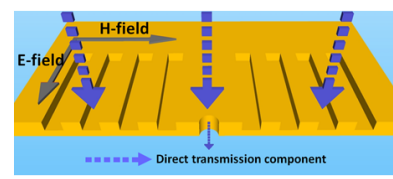

(c)

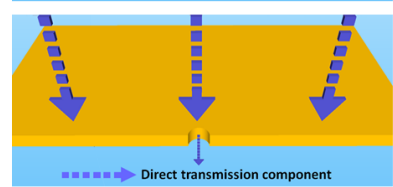

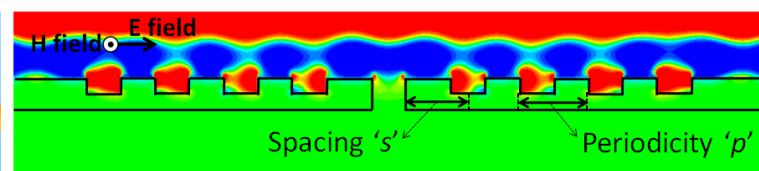

Etreldo $0 \stackrel{\mathrm{H} \text { field }}{\longrightarrow}$

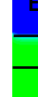

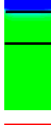

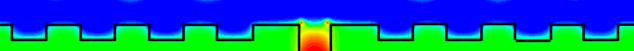

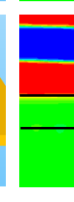

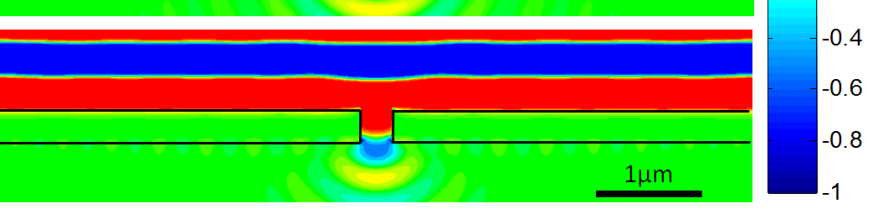

Figure 2. (a) Incoming TM polarized light can be collected and converted into the surface wave by the periodic grooves and then be recoupled into propagating light through the central hole. The SW-assisted component and the direct transmission component of the central hole can cancel each other, resulting in near-zero transmission under uniform illumination. (b) The SP coupling efficiency for TE polarized light is much smaller than that for the TM wave, and thus, the absence of interference of SP components permits significant TE polarized light transmission. (c) The transmission of a single hole without the groove pattern.

We next fabricated a number of SWEDA with linear groove pattern based on the parameters suggested by our simulation results. Fig. 3(a) shows the scanning electron microscope (SEM) image of a typical SWEDA that we have created by focused ion beam (FIB) milling. We fabricated a set of 9 SWEDAs with different spacing 's' ranging from $455 \mathrm{~nm}$ to $775 \mathrm{~nm}$. A single hole without the groove pattern was also fabricated to serve as a control. To characterize the optical properties of the SWEDA, we used a tunable wavelength laser (Spectra-Physics Tsunami Continuous wave Ti: Sapphire) as the illumination source. The transmissions through the apertures were collected by an inverted microscope with a $20 \mathrm{X}$ objective.

(a)

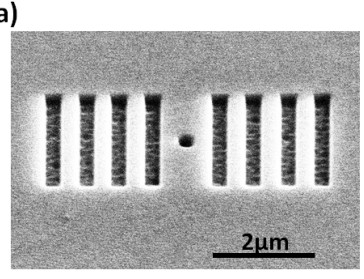

(d)

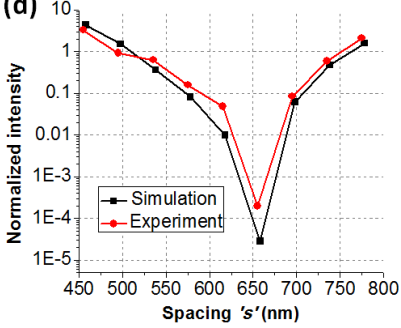

(b)

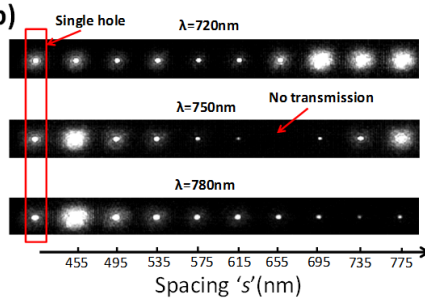

(e)

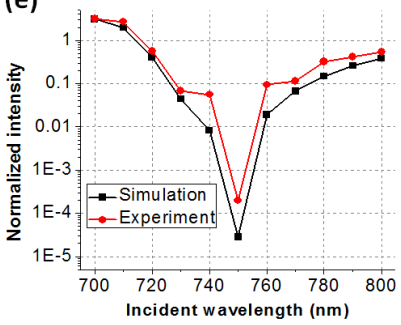

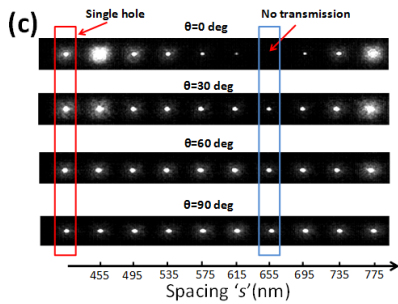

(f)

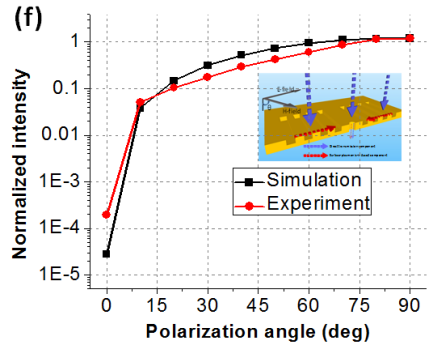

Figure 3. (a) The scanning electron microscope (SEM) image of a typical SWEDA that we have created by focused ion beam milling. (b) The optical transmission images of the 9 SWEDAs and the reference single-hole under normal-incidence illumination for three different wavelengths. (c) The optical transmission images of the 9 SWEDAs and the reference single-hole with different polarization angle. (d) The measured optical transmission signals from SWEDAs with different spacing 's' ranging from $455 \mathrm{~nm}$ to $775 \mathrm{~nm}$ (left to right). The signals from the SWEDA were normalized by that from a single hole (signal from the single hole at normal incidence was set to unity). The measured suppression factor for the optimized SWEDA is 5080. The simulated intensity is also shown for comparison. (e) The measured normalized optical transmission signals from SWEDA ( $\mathrm{s}=655 \mathrm{~nm}$ ) with different incident wavelengths. The simulation result is also shown for comparison. In this set of simulations, the permittivity values for gold at different wavelengths given in Ref. [30] were used. (f) The measured normalized optical transmission signals from SWEDA with different polarization angle. 
Fig. 3(b) shows the optical transmission images of the 9 SWEDAs and the reference single-hole at normal-incident illumination for three different wavelengths. We can see that the spacing parameter ' $\mathrm{s}$ ' does indeed have a significant impact on the transmission of the SWEDA structures. The transmission intensity measured for these SWEDAs with different spacing 's' are plotted in Fig. 3(d) (wavelength of $750 \mathrm{~nm}$ ); we used the transmission of the unadorned simple hole for normalization. The simulation prediction for each of the structures is also plotted for comparison. From the plots, the implemented SWEDA structure with s-parameter of $655 \mathrm{~nm}$ exhibited the desired near-zero transmission characteristics. The optimized SWEDA's structure parameters were a close match with our simulation predictions - the s-parameter differed by $3 \mathrm{~nm}$ (less than $0.5 \%$ ). The measured suppression factor for the optimized SWEDA was 5080. In other words, this SWEDA transmitted 5080 times less light than an unadorned simple hole of size equal to that of the central SWEDA hole. We next measured the spectral transmission response of the optimized SWEDA over a spectral range of $700 \mathrm{~nm}$ to $800 \mathrm{~nm}$. Since SWEDA's operation depends on the exact balance and opposing phase relationship of the SP-assisted transmission component and the direct transmission component, we can expect that the darkfield property of SWEDA be optimized for only a single wavelength. Fig. 3(e) shows the experimentally measured and simulation-predicted spectral transmission of the SWEDA. As expected, there is a single minimum over the range of interest and the transmission increases monotonically away from this point. It is also worth noting that the suppression factor actually remained fairly high ( $>50)$ for a bandwidth of $\sim 10 \mathrm{~nm}$. In Fig. 3(f), we show the normalized transmission of SWEDA as a function of polarization rotation angle (also see Fig. 3(c) for the optical image). The measured polarization extinction ratio is 6100, meaning that the amount of TE-transmission light through SWEDA is 6100 times that of the TM case. This is about 2 orders of magnitude larger than the previously reported results obtained by using an elliptical aperture array [31,32]. This improvement clearly demonstrates that our novel usage of destructive interference between the direct hole transmission component and SP-assisted transmission component can result in more effective nulling of the net light transmission. In turn, SWEDA in this and other forms can be used as highly sensitive detection elements.

The good agreement between the experimental and simulation spacing, wavelength and polarization angle trends, as evident in Fig. 3(d), 3(e) and 3(f), is a proof of the working principle of the proposed SWEDA. The discrepancy in extinction ratio is attributable to fabrication imperfections associated with the FIB milling process -we tend to end up with rounded structure edges experimentally. If exact matches of experiments and simulation are desired in specific applications, such imperfections may be mitigated by employing a sacrificial layer described in Ref. [33] during fabrication to help preserve the sharpness of edges.

\section{SWEDA WITH CIRCULAR GROOVE PATTERN}

The second type of SWEDA is shown in Fig. 4(a). It adopts a circular pattern for the groove design. We refer to this structure as the circular-groove based SWEDA. Due to its circularly symmetric nature, this type of SWEDA provides a polarization-independent behavior for signal detection and imaging.

(a)

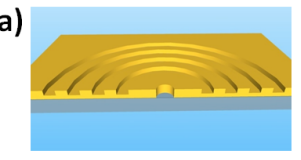

(b)

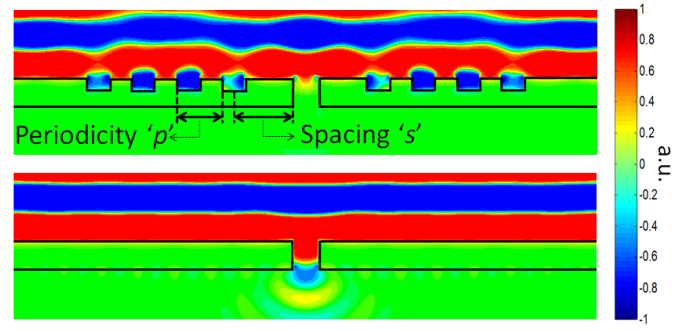

Figure 4. (a) The simulation for the SWEDA structure with a circular pattern. (b) The simulation of a single hole without the groove pattern.

The simulation program allowed us to map out the interplay of these parameters and the overall SWEDA system characteristics. We were able to arrive at a design parameter set (Fig. 4(a)) that provides a suppression factor of 6640 by the simulation program. The simulated electric-field distributions for this particular SWEDA design (Fig. 4(a)) and that of a corresponding simple hole (Fig. 4(b)) are shown here. We can see that the SWEDA structure should indeed be able to suppress light transmission through the central hole significantly. 
We next fabricated a number of SWEDA structure based on the parameters suggested by our simulation results. Fig. 5(a) shows the scanning electron microscope (SEM) image of a typical SWEDA that we have created by FIB milling. We fabricated a set of 13 SWEDAs with different spacing 's' ranging from $540 \mathrm{~nm}$ to $1020 \mathrm{~nm}$. A single hole without the groove structure was also fabricated to serve as a control.

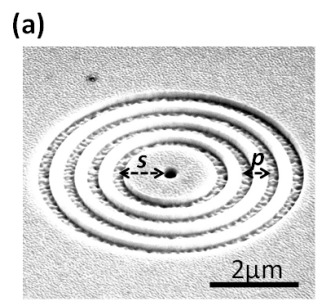

(c)

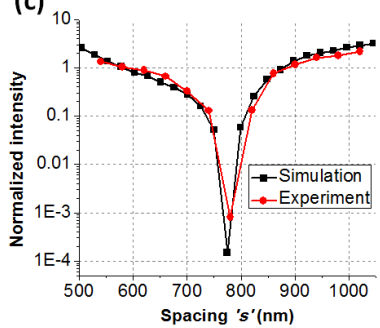

(b)

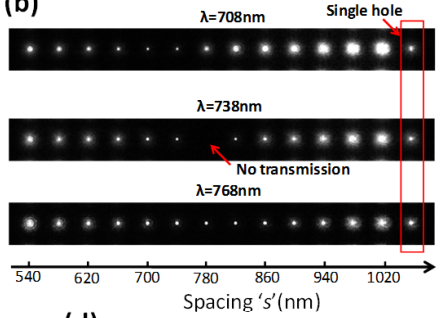

(d)

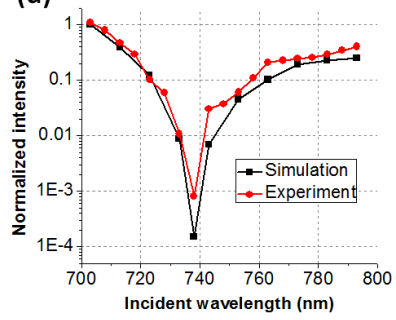

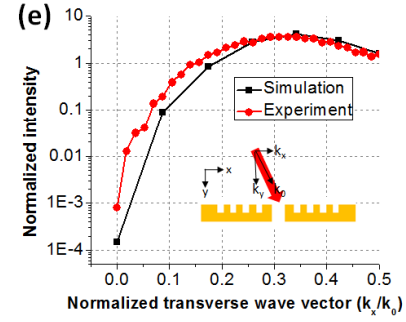

Figure 5. Experimental characterization of the linear-groove based SWEDA. (a) The SEM image of a typical fabricated SWEDA. (b) The optical transmission images of the 9 SWEDAs and the reference single-hole under normal-incidence illumination for three different wavelengths. (c) The measured optical transmission signals from SWEDAs with different spacing ' $s$ ' ranging from $455 \mathrm{~nm}$ to $775 \mathrm{~nm}$ (left to right). The signals from the SWEDA were normalized by that of asingle hole (signal from the single hole was set to unity). The measured suppression factor for the optimized SWEDA is 5080. The simulated intensity is also shown for comparison. (d) The measured normalized optical transmission signals from SWEDA $(s=655 \mathrm{~nm})$ with different incident wavelengths. (e) The optical transmission images of the SWEDAs for different polarization angles at $\lambda=750 \mathrm{~nm}$ (left). The normalized intensity is plotted as a function of polarization angle (right). The measured polarization extinction ratio for TE and TM incidence is 6100 .

Fig. 5(b) shows the optical transmission images of the 13 SWEDAs and the reference single-hole at normal incidence for three different wavelengths. We can see that the spacing parameter ' $s$ ' does indeed have a significant impact on the transmission of the SWEDA structures. The transmission intensity measured for these SWEDA structures are plotted in Fig. 5(c) (wavelength of $738 \mathrm{~nm}$ ); we used the unadorned simple hole for normalization. The simulation prediction for each of the structures is also plotted for comparison. From the plots, the implemented SWEDA structure with sparameter of $780 \mathrm{~nm}$ exhibited the desired near-zero transmission characteristics. The optimized SWEDA's structure parameters were a close match with our simulation predictions - the s-parameter differed by $6 \mathrm{~nm}$ (less than $0.8 \%$ ). The measured suppression factor for the optimized SWEDA was 1230. In other words, this SWEDA transmitted 1230 times less light than an unadorned simple hole of size equal to that of the central SWEDA hole.

We next measured the spectral response of the optimized SWEDA over a spectral range of $700 \mathrm{~nm}$ to $790 \mathrm{~nm}$. Fig. 5(d) shows the experimentally measured and simulation-predicted spectral transmission of the SWEDA. As expected, there is a single minimum over the range of interest and the transmission increased monotonically away from this point. It is also worth noting that the suppression factor actually remained fairly high $(>50)$ for a bandwidth of $\sim 10 \mathrm{~nm}$.

For a given incident light field, we can decompose it into different plane wave components with respect to the transverse wave vector [34-36]. In Fig. 5(e), we measure the transmission of the SWEDA as a function of the normalized transverse wave vector. Fig. 5(e) acts as a system transfer function of the SWEDA: it rejects the normal incident plane wave component and captures the high spatial frequency component that contains information about the scatterer. 


\section{DEMONSTRATION OF SWEDA'S ABILITY TO BOOST DETECTION SENSITIVITY}

In order to demonstrate of SWEDA's ability to boost detection sensitivity, we next simulated the translation of a cylindrical dielectric object (radius $300 \mathrm{~nm}$, thickness $200 \mathrm{~nm}$, displacement height $300 \mathrm{~nm}$, permittivity 2.25) across the top of the SWEDA structure (Fig. 6(a)- 6(c)). We can see that the SWEDA began to transmit light significantly when the object's presence directly above the central hole significantly perturbed the direct transmission component and, consequently, disrupted the delicately balanced destructive interference condition. We further observed that the presence of the object above the groove structures did perturb the SWEDA to a certain extent as well. However, the impact was much less significant (Fig. 6(d)); this can be well appreciated by noting that the generation of the surface wave occurred over the entire area associated with the ring grooves, so the localized changes of the light field over the smaller area had a diminished impact on the overall surface wave component. As a whole, this simulation indicates that the SWEDA is maximally sensitive to light field heterogeneity directly above the central hole.

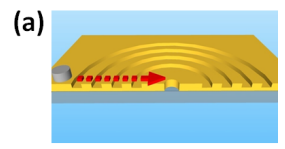

(b)

(c)
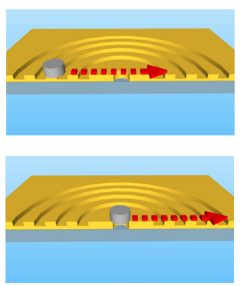
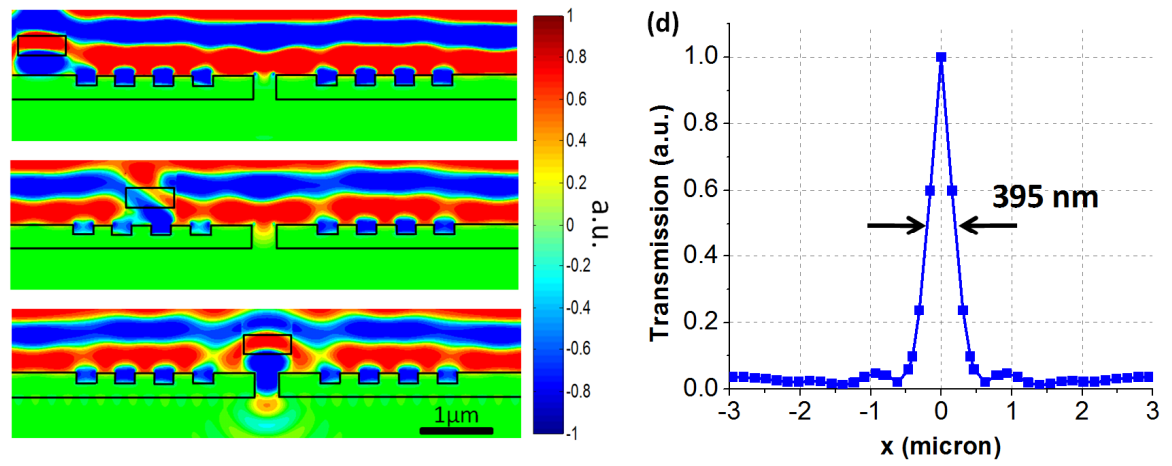

Figure 6. (a-c) Simulations of a cylindrical scatterer (radius $300 \mathrm{~nm}$, thickness $200 \mathrm{~nm}$, displacement height 300 $\mathrm{nm}$, permittivity 2.25) translating across the SWEDA. (d) The transmission signal curve from the SWEDA as the cylindrical scatterer (the same as (a)-(e)) moves across it. The full width at half maximum was determined to be $395 \mathrm{~nm}$.

The ability of such a SWEDA to improve small signal detection is illustrated in the following experiment. We prepared a sample comprised of an ITO-coated glass slide that was marked with shallow pits of radius of $175 \mathrm{~nm}$ and $250 \mathrm{~nm}$ via the FIB (Fig. 7(a) and (b)). Next, we transmitted a uniform light field of intensity about $0.2 \mathrm{~W} / \mathrm{cm}^{2}$ from a $738 \mathrm{~nm}$ laser through the sample. We then used a 1:1 relay microscope to project a virtual image of the pits onto our optimized circular-groove based SWEDA. We next raster-scanned the sample and measured the light transmission through the SWEDA at each point of the scan. We then generated an image of the sample from the collected data. As is evident in Fig. 7(c) and (d), SWEDA allowed us to identify the presence of the two pits with little difficulty. We next acquired images of the same pits (Fig. 7(e) and (f)) with a simple camera (based on the same sensor chip). It is difficult to identify the presence of the two pits in this case. The total light fluence incident on the sample for both the SWEDA and camera image acquisitions was kept the same to allow for direct result comparisons.

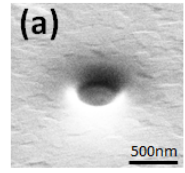

(b)

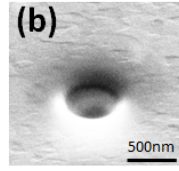

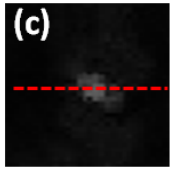

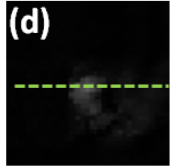

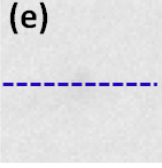

(f)

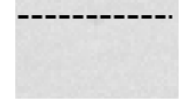

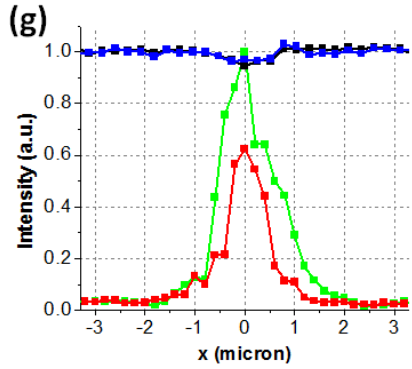

Figure 7. The sensitivity enhancement demonstration for the circular-groove based SWEDA. (a) and (b) The SEM images of the $175 \mathrm{~nm}$ and $250 \mathrm{~nm}$ pits on the ITO coated glass. (c) and (d) The SWEDA-based raster-scanned images of the samples (a) and (b). (e) and (f) Microscope images of the samples (a) and (b) under the same illumination condition as the SWEDA collected images using a conventional camera with the same CMOS chip. (g) Center line traces of the images in (c)-(f). Please see (C-F) for color reference guide. The observed image contrast (signal / background) enhancement is $\sim 25 \mathrm{~dB}$ for the $175 \mathrm{~nm}$ pit and $\sim 27 \mathrm{~dB}$ for the $250 \mathrm{~nm}$ pit. 
Fig. 7(g) shows plots of signal traces across the images. The SWEDA-acquired data were normalized on the same scale. The camera image data were normalized versus the average background signal. The backgrounds associated with the SWEDA-acquired data were low and the contributive signals from the pits were well discernible. In fact, the contributive signals were sufficiently well resolved that we can use them to quantify their relative strengths for the two pits. In comparison, the high backgrounds in the camera images combined with the associated noise masked the scattering contributions from the pits. The measured contrast improvement was $25 \mathrm{~dB}$ for the $175 \mathrm{~nm}$ pit and $27 \mathrm{~dB}$ for the $250 \mathrm{~nm}$ pit.

\section{DARKFIELD IMAGING BY SWEDA}

As pointed out in our introduction, circular-groove based SWEDA can potentially be employed to perform darkfield microscopy imaging on the sensor level. The principle involved is substantially different from that of a conventional darkfield microscope. While a conventional system depends on oblique illumination and a relatively small objective angle of collection to screen out the uniform background via a fairly sophisticated and bulky optical arrangement, the ability of circular-groove based SWEDA to screen out uniform background presents a more direct approach. To demonstrate that such a system can indeed be implemented, we employed our optimized circular-groove based SWEDA in the same experimental scheme to scan slides of starfish embryos in different developmental stages. The illumination intensity was $0.2 \mathrm{~W} / \mathrm{cm}^{2}$. Fig. 8 (a) and (d) show the results. Similar images of the specimens taken with a standard microscope are shown in Fig. 8(c) and (f) for comparison. We can see that the SWEDA generated image has a dark background, as is consistent with a darkfield microscope image. We can also see that the edge and interior of the starfish embryo appeared brighter in the SWEDA image and darker in the control image. This is again consistent with our expectations of a darkfield image as sample locations with substantial scattering should appear brighter in a darkfield image and darker in a simple transmission image. We would like to emphasize that this is a proof-of-concept experiment. A feasible darkfield microscope can be implemented by employing a laser as the light source in a standard microscope and using a sensor chip patterned with a grid of tightly spaced circular-groove based SWEDA as the microscope camera.
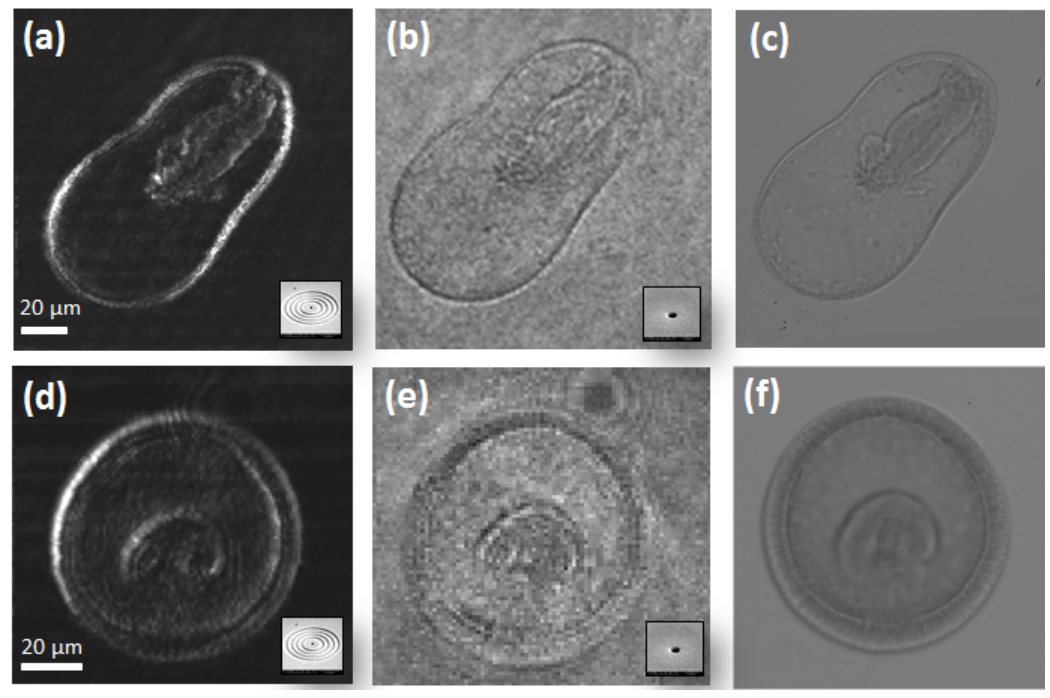

Figure 8. The demonstration of darkfield imaging by SWEDA. (a) and (d) The SWEDA-based raster-scanned images of the starfish embryos. (b) and (e) The single-hole-based raster-scanned images of the starfish embryos. (c) and (f) Conventional bright field microscope images.

\section{IMPROVING WEAK SIGNAL IDENTIFICATION BY A SWEDA PIXEL}

We also fabricated a SWEDA pixel prototype [37] on a commercial CMOS imager sensor (Aptina MT9P031, with a 2.2 $\mu \mathrm{m}$ pixel size), as shown in Fig. 9. Fig. 9(a) shows the pixel of the CMOS image sensor. We first spin-coated a $150 \mathrm{~nm}$ 
thick poly methyl methacrylate (PMMA) layer onto the microlens array of the image sensor to planarize the surface, and then coated a $330 \mathrm{~nm}$ gold layer using a thermal evaporator (a $5 \mathrm{~nm}$ chromium layer was used as an adhesion layer). After the metal coating, we used focused ion beam (FEI Nova200 dual-beam system) to mill the SWEDA structure. We chose a central hole diameter of $500 \mathrm{~nm}, \mathrm{p}=676 \mathrm{~nm}, \mathrm{~s}=690 \mathrm{~nm}$, and depth of groove $=190 \mathrm{~nm}$. Fig. 9(c) shows the focused ion beam image of a typical structure. In Fig. 9(a), the individual pixel can be barely seen due to the fact that only a thin planarized layer is used and the topology of the surface is not absolutely flat. Fig. 9(d) shows the image taken by the sensor under a uniform light illumination with three different wavelengths. The measured best suppression ratio was $\sim 1100$ at $775 \mathrm{~nm}$ (Fig. 9(d)).

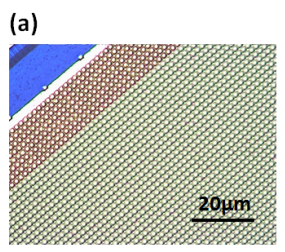

(c)

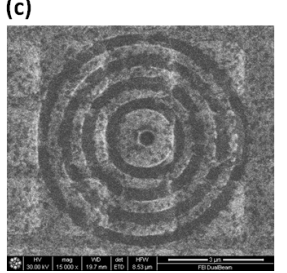

(b)
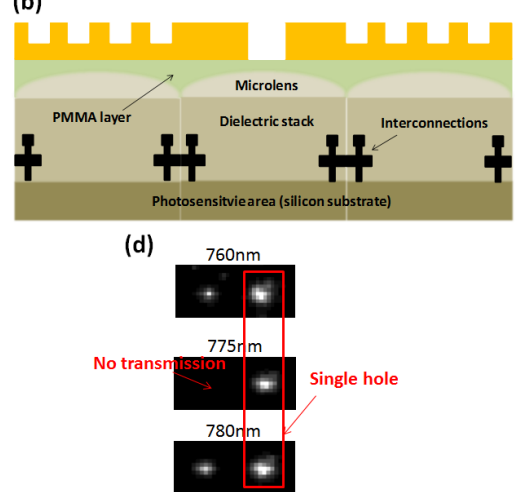

Figure 9. (a) The pixel of the CMOS image sensor. (b) The scheme of SWEDA pixel. (c) The FIB image of the SWEDA structure. (d) Images taken by the sensor under a uniform light illumination with three different wavelengths. The SWEDA pixel is optimized for a single wavelength, and the measured best suppression ratio was $\sim 1100$ at $775 \mathrm{~nm}$.

Next, we conducted an experiment to characterize the SNR of the SWEDA pixel under a high background. We used a Ti-Sapphire laser as the background illumination source (wavelength of $775 \mathrm{~nm}$ ) and a focused He-Ne laser $(632 \mathrm{~nm})$ to generate a spatially localized signal beam (10X, $0.22 \mathrm{NA}$ objective lens). The signal beam focus had a diameter of $\sim 2 \mu \mathrm{m}$ and was, thus, a match to a single sensor pixel size. We define the SBR (signal-to-background ratio) as the ratio of the signal intensity to background intensity. In our experiment, the background intensity was $3.96 \mathrm{~W} / \mathrm{m}^{2}$. Fig. 10 (a) shows the SNR (mean value / standard deviation of the signal) versus different SBR values. For each data point, we collected 1000 consecutive frames and used this data to calculate the SNR (the exposure time is $5 \mathrm{~ms}$ for each frame). We see that the overall SNR performance of the SWEDA pixel was better than that of single undressed pixel over a significant SBR range. At the small signal region of Fig. 10(a), the SNR of SWEDA pixel was higher than that of the single pixel since the noise fluctuation of the bright background was the dominant noise term at this region. At the large signal region of Fig. 3(a), the SNR of single pixel became similar or exceeded that of the SWEDA pixel because the noise from the signal itself began to take over. At this point, the much larger photon acceptance area of the single undressed pixel versus the SWEDA pixel (experimentally measured be to a ratio of $\sim 22$ ) provided an overriding advantage.

A more interesting demonstration is the SWEDA pixel's ability to provide SNR $>1$ for a signal with a very low SBR value, a challenge that has not been met by conventional detection approaches. Here, we used an optical chopper to modulate the input focus signal beam (the optical chopper is like a switch to turn the signal beam on and off at a certain rate) and recorded the time trace of the pixel readout in Fig. 10(b). The control case with single pixel is shown in Fig. 10(b) for comparison. We see that, for the single pixel in Fig. 10(b), the signal was totally masked by the background $(\mathrm{SNR}=0.26)$; yet for the SWEDA, it is well resolvable $(\mathrm{SNR}=2.2)$. Therefore, for the weak signal buried in high background scenarios, our SWEDA pixel approach does provide a better platform than the conventional detection approaches. 
(a)

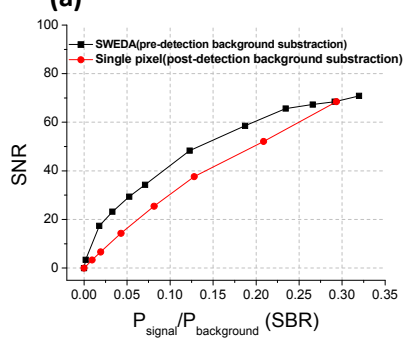

(b)

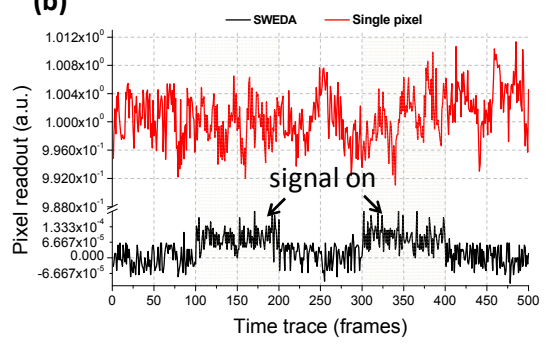

Figure 10. (a) The SNR versus different SBR values for single un-patterned pixel and SWEDA pixel. (b) SWEDA pixel's ability to provide SNR $>1$ for a signal with a very low SBR value.

\section{BIOTIN-STREPTAVIDIN BIOSENSING WITH SWEDA}

Another application of SWEDA is for highly sensitive biosensing experiments. In particular, the widely used biotinstreptavidin reaction could be adapted for use with SWEDA for the optical detection of streptavidin tagged molecules of interest, according to preliminary experiments and simulations. In this detection scheme, biotin is placed on the gold surface layer of the SWEDA. As a solution containing the corresponding streptavidin molecules brings these molecules into contact with the biotin-coated surface of the SWEDA, streptavidin molecules will bind to the biotin molecules, causing a local change in the index of refraction on the surface of the SWEDA structure. This change in the index of refraction will chance the surface wave component of light, disturbing the delicate destructive interference condition and allowing light to be transmitted through. Hence, the presence of the streptavidin-tagged molecules would be converted into an optical signal by the SWEDA.

To explore this concept, we conducted a simulation using CST Microwave Studio again. We first optimized for a new set of parameters for the SWEDA structure to operate in water (refractive index 1.33). Next, to simulate streptavidin molecules, about $5 \mathrm{~nm}$ in size with refractive index 1.45 [38], we conducted another simulation with a $5 \mathrm{~nm}$ thick layer of streptavidin covering the entire surface. As you can see in Fig. 11, the presence of the streptavidin on the surface of the SWEDA breaks the deconstructive interference condition, allowing for much greater light transmission. The transmission ratio shown in Fig. 11(b) and 11(c) is about 1000. These simulation results demonstrate the feasibility of using SWEDA in biosensing applications with biotin and streptavidin.

(a)

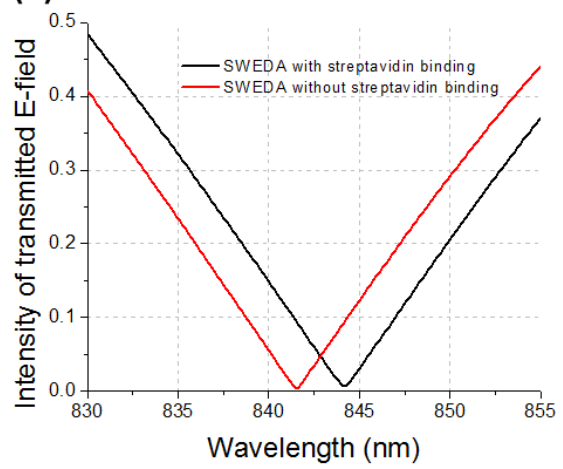

(b)

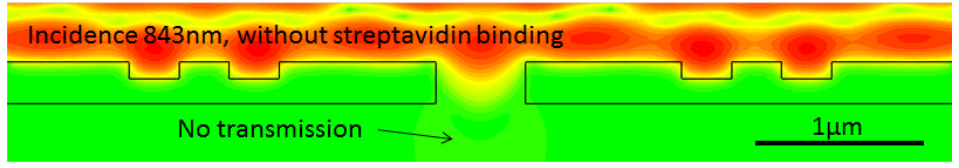

(c)

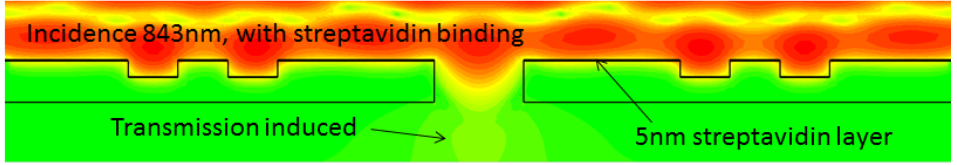

Fig. 11 (a) The spectrum of intensity of transmitted E-field (the intensity of incident E-field is set to be 1). (b) The E-field intensity distribution without streptavidin binding. (c) The E-field intensity distribution with $5 \mathrm{~nm}$ streptavidin binding.

Compared with the conventional surface-plasmon-resonance (SPR) sensor, the proposed platform does not employ the Kretschmann configuration and is based on direct coupling of normally incident radiation, making this scheme very sensitive to localized changes in refractive index in the vicinity of the metallic surface of the structure. The sensing 
approach described here does not require any prism coupling mechanism, thereby making the miniaturization of these sensors feasible.

\section{DISCUSSION}

As our experimental findings indicate, SWEDA is a robust, structurally simple and highly compact approach to accomplish optical background suppression and/or polarized light field suppression. It is also worth noting that, in principle, there is no theoretical limit to how close the SWEDA darkfield suppression factor can approach infinity; the practical limit is only set by the fabrication tolerance and the net transmission through the opaque metal layer.

SWEDA technology can potentially be used in a range of different applications. The linear-groove based SWEDA demonstrated in the present work is a highly-compact and highly-sensitive polarization sensor. Since the polarization state of light will change during the interaction with chiral materials, this SWEDA design may also find some applications in on-chip detection of some chiral materials such as sugar, proteins and DNA [39, 40]. The ability to fully suppress a coherent background as exhibited by the circular-groove based SWEDA can be useful for small signal detection in metrology applications. It is especially applicable in detection scenarios where the overall background intensities fluctuate with time. As our background subtraction occurs at the individual pixel level, SWEDA technology removes the need for balanced detection schemes. The pre-detection background subtraction, which is a light cancellation process, is also intrinsically more sensitive than post-detection cancellation schemes that are susceptible to intrinsic detection statistical variations. The inclusion of chemical reagents in the central hole can also turn such a SWEDA structure into a high-sensitivity sensor that can react to small refractive index changes of the reagents.

SWEDA structures can be fabricated directly on top of CCD or CMOS sensor pixels. The small size and planar design of SWEDA make such implementation particularly suited for foundry fabrication. Sensor chips with broad-bandwidth SWEDA can then be used in place of the standard camera sensor to accomplish sensor level darkfield imaging. Such systems, in combination with a coherent light source, can transform a standard microscope into a simpler and cheaper darkfield microscope than current darkfield microscopes. Such systems can also enable edge-detection imaging in machine vision applications if the illumination source employed is coherent. A SWEDA array can also replace the hole array in the optofluidic microscope (OFM) $[1,2,41]$ - a low-cost, lensless and high resolution microscopy approach, to accomplish darkfield microscopy imaging on a chip. The use of SWEDA in this case is especially appropriate as both the OFM and SWEDA implementation are well suited for semiconductor mass-fabrication. In fact, it is difficult to envision a more compact and cost-effective approach for incorporating darkfield imaging abilities in an OFM system.

Finally, we would like to note that the general concept of exactly balancing the surface-wave-induced component and direct light transmission component in a destructive interference manner is a novel idea that can inspire other surfacewave-structures with novel properties. Effectively, such structures are tiny interferometers ( $\sim 6$ microns or less) that can be fabricated on a single metal substrate and which have excellent stability (our SWEDA structures exhibited no significant performance drift over the entire duration of our experiments). Since the structure is planar, it can be mass produced in a semiconductor foundry. The proposed structure can also be redesigned for operation at longer wavelengths. As an example of other potential applications, we believe that the concept of SWEDA can be applied to optical isolation in a ultra-compact format, polarization control in semiconductor lasers [42], wavefront detection, extending depth of field of the type II aperture-based imaging device [43], and perspective imaging [44] by customizing the optical transfer function on the pixel level.

\section{REFERENCES}

[1] X. Cui, L. Lee, X. Heng et al., "Lensless high-resolution on-chip optofluidic microscopes for Caenorhabditis elegans and cell imaging," Proceedings of the National Academy of Sciences, 105(31), 10670 (2008).

[2] G. Zheng, S. A. Lee, S. Yang et al., "Sub-pixel resolving optofluidic microscope for on-chip cell imaging," Lab on a Chip, 10(22), 3125-3129 (2010).

[3] H. Lee, Y. Liu, D. Ham et al., "Integrated cell manipulation system -- CMOS/microfluidic hybrid," Lab on a Chip, 7(3), 331-337 (2007). 
[4] P. Swanson, R. Gelbart, E. Atlas et al., "A fully multiplexed CMOS biochip for DNA analysis," Sensors \& Actuators: B. Chemical, 64(1-3), 22-30 (2000).

[5] J. Wu, X. Cui, G. Zheng et al., "Wide field-of-view microscope based on holographic focus grid illumination," Optics letters, 35(13), 2188-2190 (2010).

[6] F. Heer, S. Hafizovic, W. Franks et al., "CMOS microelectrode array for bidirectional interaction with neuronal networks," IEEE Journal of Solid State Circuits, 41(7), 1620 (2006).

[7] T. Ebbesen, H. Lezec, H. Ghaemi et al., "Extraordinary optical transmission through sub-wavelength hole arrays," Nature, 391(6668), 667-669 (1998).

[8] T. Thio, K. M. Pellerin, R. A. Linke et al., "Enhanced light transmission through a single subwavelength aperture," Opt. Lett., 26(24), 1972-1974 (2001).

[9] H. Lezec, A. Degiron, E. Devaux et al., "Beaming light from a subwavelength aperture," Science, 297(5582), 820-822 (2002).

[10] T. Thio, H. Lezec, T. Ebbesen et al., "Giant optical transmission of sub-wavelength apertures: physics and applications," Nanotechnology, 13(3), 429-432 (2002).

[11] H. Lezec, and T. Thio, "Diffracted evanescent wave model for enhanced and suppressed optical transmission through subwavelength hole arrays," Optics express, 12(16), 3629-3651 (2004).

[12] R. Hollingsworth, and R. Collins, [Plasmon enhanced near-field optical probes] Google Patents, (2005).

[13] H. Schouten, N. Kuzmin, G. Dubois et al., "Plasmon-assisted two-slit transmission: Young's experiment revisited," Physical Review Letters, 94(5), 53901 (2005).

[14] L. Chen, J. Robinson, and M. Lipson, "Role of radiation and surface plasmon polaritons in the optical interactions between a nano-slit and a nano-groove on a metal surface," Optics Express, 14(26), 12629-12636 (2006).

[15] G. Gay, O. Alloschery, B. Viaris de Lesegno et al., "The optical response of nanostructured surfaces and the composite diffracted evanescent wave model," Nat Phys, 2(4), 262-267 (2006).

[16] P. Lalanne, and J. Hugonin, "Interaction between optical nano-objects at metallo-dielectric interfaces," Nature Physics, 2(8), 551 (2006).

[17] L. Aigouy, P. Lalanne, J. Hugonin et al., "Near-field analysis of surface waves launched at nanoslit apertures," Physical Review Letters, 98(15), 153902 (2007).

[18] D. Pacifici, H. Lezec, and H. Atwater, "All-optical modulation by plasmonic excitation of CdSe quantum dots," Nature photonics, 1(7), 402-406 (2007).

[19] A. Drezet, C. Genet, and T. Ebbesen, "Miniature plasmonic wave plates," Physical Review Letters, 101(4), 43902 (2008).

[20] E. Laux, C. Genet, T. Skauli et al., "Plasmonic photon sorters for spectral and polarimetric imaging," Nature Photonics, 2(3), 161-164 (2008).

[21] H. Liu, and P. Lalanne, "Microscopic theory of the extraordinary optical transmission," Nature, 452(7188), 728-731 (2008).

[22] D. Pacifici, H. Lezec, L. Sweatlock et al., "Universal optical transmission features in periodic and quasiperiodic hole arrays," Optics Express, 16(12), 9222-9238 (2008).

[23] B. Ung, and Y. Sheng, "Optical surface waves over metallo-dielectric nanostructures: Sommerfeld integrals revisited," Optics Express, 16(12), 9073-9086 (2008).

[24] G. Gbur, H. Schouten, and T. Visser, "Achieving superresolution in near-field optical data readout systems using surface plasmons," Applied Physics Letters, 87, 191109 (2005).

[25] S. Maier, [Plasmonics: fundamentals and applications] Springer Verlag, (2007).

[26] D. Pacifici, H. Lezec, H. Atwater et al., "Quantitative determination of optical transmission through subwavelength slit arrays in Ag films: Role of surface wave interference and local coupling between adjacent slits," Physical Review B, 77(11), 115411 (2008).

[27] G. Zheng, X. Cui, and C. Yang, "Surface-wave-enabled darkfield aperture for background suppression during weak signal detection," Proceedings of the National Academy of Sciences, 107(20), 9043 (2010).

[28] CST Microwave Studio 2009 by Computer Simulation Technology [Online at http://www.cst.com/].

[29] G. Boisset, "Luxpop-Thin film and bulk index of refraction and photonics calculations." [Online at http://www.luxpop.com/].

[30] E. Palik, and G. Ghosh, [Handbook of optical constants of solids] Academic press, (1985).

[31] J. Elliott, I. I. Smolyaninov, N. I. Zheludev et al., "Wavelength dependent birefringence of surface plasmon polaritonic crystals," Physical Review B, 70(23), 233403 (2004). 
[32] R. Gordon, A. Brolo, A. McKinnon et al., "Strong polarization in the optical transmission through elliptical nanohole arrays," Physical review letters, 92(3), 37401 (2004).

[33] J. Leen, P. Hansen, Y. Cheng et al., "Improved focused ion beam fabrication of near-field apertures using a silicon nitride membrane," Optics Letters, 33(23), 2827-2829 (2008).

[34] J. Kong, [Electromagnetic wave theory] EMW publishing, (2005).

[35] G. Zheng, Y. Wang, and C. Yang, "Pixel level optical-transfer-function design based on the surface-waveinterferometry aperture," Opt. Express, 18(16), 16499-16506 (2010).

[36] Y. Wang, G. Zheng, and C. Yang, "Characterization of acceptance angles of small circular apertures," Opt. Express, 17, 23903-23913 (2009).

[37] G. Zheng, and C. Yang, "Improving weak-signal identification via predetection background suppression by a pixel-level, surface-wave enabled dark-field aperture," Optics letters, 35(15), 2636-2638 (2010).

[38] K. Caswell, J. Wilson, U. Bunz et al., "Preferential End-to-End Assembly of Gold Nanorods by BiotinStreptavidin Connectors," J. Am. Chem. Soc, 125(46), 13914-13915 (2003).

[39] G. Fasman, [Circular dichroism and the conformational analysis of biomolecules] Plenum Pub Corp, (1996).

[40] K. Minakawa, H. Yamada, K. Sasagawa et al., "Microchamber Device Equipped with Complementary Metal Oxide Semiconductor Optical Polarization Analyzer Chip for Micro Total Analysis System,” Jpn. J. Appl. Phys., 48(4), 04C192 (2009).

[41] X. Heng, D. Erickson, L. Baugh et al., "Optofluidic microscopy ---- method for implementing a high resolution optical microscope on a chip," Lab on a Chip, 6(10), 1274-1276 (2006).

[42] N. Yu, Q. Wang, C. Pflugl et al., "Semiconductor lasers with integrated plasmonic polarizers," Applied Physics Letters, 94, 151101 (2009).

[43] X. Heng, X. Cui, D. Knapp et al., "Characterization of light collection through a subwavelength aperture from a point source," Optics express, 14(22), 10410-10425 (2006).

[44] R. Ng, M. Levoy, M. Bredif et al., "Light field photography with a hand-held plenoptic camera," Computer Science Technical Report CSTR, 2, (2005). 